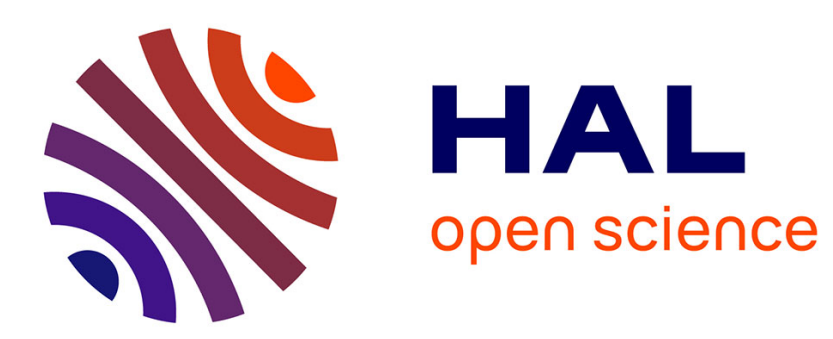

\title{
Particle alignment and clustering in sheared granular materials composed of platy particles
}

\author{
Mauricio Boton, Nicolas Estrada, Emilien Azéma, Farhang Radjai
}

\section{To cite this version:}

Mauricio Boton, Nicolas Estrada, Emilien Azéma, Farhang Radjai. Particle alignment and clustering in sheared granular materials composed of platy particles. European Physical Journal E: Soft matter and biological physics, 2014, 37, 8p. 10.1140/epje/i2014-14116-0 . hal-01084809

\section{HAL Id: hal-01084809 \\ https://hal.science/hal-01084809}

Submitted on 20 Nov 2014

HAL is a multi-disciplinary open access archive for the deposit and dissemination of scientific research documents, whether they are published or not. The documents may come from teaching and research institutions in France or abroad, or from public or private research centers.
L'archive ouverte pluridisciplinaire HAL, est destinée au dépôt et à la diffusion de documents scientifiques de niveau recherche, publiés ou non, émanant des établissements d'enseignement et de recherche français ou étrangers, des laboratoires publics ou privés. 


\title{
Particle alignment and clustering in sheared granular materials composed of platy particles
}

\author{
Mauricio Boton ${ }^{1}$, Nicolas Estrada ${ }^{1}$, Emilien Azéma ${ }^{2}$, and Farhang Radjaï ${ }^{2}$ \\ 1 Departamento de Ingeniería Civil y Ambiental - CeiBA Complex Systems Research Center, Universidad de Los Andes, Bogotá, \\ Colombia \\ 2 Université Montpellier 2, CNRS, LMGC, Place Eugène Bataillon, 34095 Montpellier cedex 05, France
}

Received: date / Revised version: date

\begin{abstract}
By means of molecular dynamics simulations, we investigate the texture and local ordering in sheared packings composed of cohesionless platy particles. The morphology of large packings of platy particles in quasistatic equilibrium is complex due to the combined effects of local nematic ordering of the particles and anisotropic orientations of contacts between particles. We find that particle alignment is strongly enhanced by the degree of platyness and leads to the formation of face-connected clusters of exponentially decaying size. Interestingly, due to dynamics in continuous shearing, this ordering phenomenon emerges even in systems composed of particles of very low platyness differing only slightly from spherical shape. The number of clusters is an increasing function of platyness. However, at high platyness the proportion of face-face interactions is too low to allow for their percolation throughout the system.
\end{abstract}

PACS. PACS-key discribing text of that key - PACS-key discribing text of that key

\section{Introduction}

In many particulate materials found in nature and industry, scientists and engineers need to quantify the effect of complex particle shapes. This is of major importance in the context of civil engineering and powder technology, where most processes need to be optimized or revised following the dramatic degradation of natural resources. For instance, elongated and platy particles occur in pharmaceutical products, angular shaped particles are common in rocks and soils, and nonconvex particles can be found in metallurgical and sintered powders $[1,2]$. The grain shape considerably affects the quasi-static mechanical behavior of granular materials $[4-9,14,33]$. There is a multitude of potential particle morphologies and recently a wide range of nontrivial effects were - systematically - reported by means of discrete numerical simulations $[10-17,19]$. Indeed, one of the major interests of discrete approach is to make it possible to control precisely the shape of the particles with continuously-variables parameters.

Most evident effects of particle shape concern the packing fraction and shear strength of granular materials. For instance, packings of elongated $[10-12,15]$ and nonconvex $[20,21]$ particles have unusually high or low packing fractions, while the shear strength is observed to grow as the shape increasingly deviates from the disk or the sphere. The shear strength in packing of polyhedral particles increases with angularity and saturates at a maximum, whereas the packing fraction declines towards a plateau $[16,19]$.
Such remarkable properties of packings composed of nonspherical particles are closely related to their specific disorder induced essentially by steric exclusions and the force balance condition for each particle. The contact networks resulting from various shapes appear to be highly complex and hardly amenable to simple statistical modeling. For example, face/face interactions are critical for polyhedra whereas interlocking of the particles is important in the case of non-convex shapes $[15,21,16,18,19]$. In the same way, nematic order appears in packings of elongated particles $[10-12,9,15,18]$. More generally, packings composed of shape-anisotropic particles (i.e. elongated/prolate or flattened/oblate) have attracted increasing interest in recent years [22]. Oblate particles prefer facing along the major principal stress direction with the flat side, while prolate particles prefer orientation of their long axis along the minor principal stress direction [2328]. It is also shown that, with increasing particle aspect ratio, the weak force network transforms from a passive stabilizing agent with respect to strong force chains to an active force-transmitting network for the whole system [18].

Nevertheless, it appears that the focus of most systematic investigations of particle anisotropic shape effects has essentially been concerned elongated particles while the effect of platy shape remains still poorly investigated. Such particles occur, for example, in clayey soils, where the local ordering is generally attributed to the cohesive interactions between particles [32-34]. 


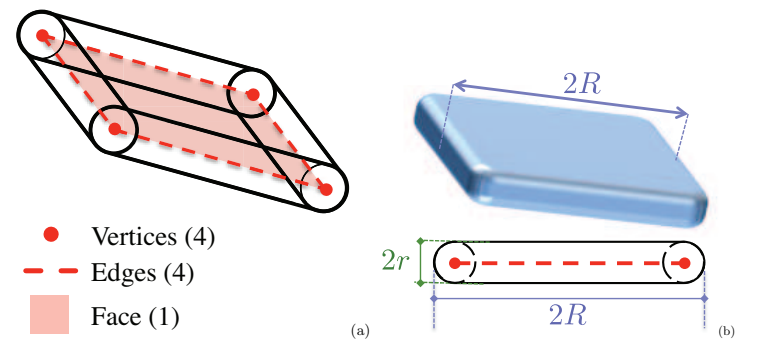

Fig. 1. (a) Scheme of a spheroplate. (b) Definition of the maximum and minimum radii, $R$ and $r$ respectively.

In this paper, we are interested in the texture and local order in numerically sheared packings composed of platy particles in three dimensions with increasing aspect ratio. We focus on the particle orientations, particle-particle interactions, and the emergence of locally ordered structures involving face-face contacts between particles. As we shall see below, the spontaneous alignment of the particles is accompanied by a strong increase of the proportion of face-face interactions leading to the formation of face-connected clusters. These clusters are characterized in terms of their number and size distributions. It is remarkable that this ordering phenomenon emerges solely as a consequence of particle shape even in the absence of cohesion.

Our numerical setup is identical to that used for the investigation of shear strength and volume-change behavior in a previous paper by the same authors as a function of the degree of platyness of the particles [31]. The samples are composed of square plates, which we approximate as spheropolyhedra $[29,8,30]$ and simulated by means of the Molecular Dynamics (MD) method. In the following, we introduce the numerical model in Sec. II. In Sec. III, we present our results and, finally, in Sec. IV, we end with a summary of the most salient results and a brief discussion.

\section{Numerical model}

\subsection{Platy particles}

The particles are square plates with rounded edges, built as spheropolyhedra resulting from sweeping a sphere around a polyhedron. Mathematically, this corresponds to a Minkows addition of a sphere with a polyhedron (for some examples of aspherical particles built as spheropolyhedra, see Refs. [35] and [29] for cylinders; Ref. [36] for particles with shapes as complex as that of a cow; Ref. [8] for cylinders, tetrahedra, and intersecting cylinders; and Ref. [37] for irregular polyhedra). Specifically, our particles are spheroplates resulting from a Minkowsky addition of a square plate and a sphere. Each particle has three features: four vertices, four edges, and one plane ; Fig.1(a).

The platyness $\eta$ of these spheroplates is defined as

$$
\eta=\frac{R-r}{R}
$$

where $R$ and $r$ are, respectively, the maximum and minimum radii of the spheroplate as defined in Fig.1(b) $(r$ is also called the spheroradius of the spheroplate). Note that $\eta$ varies from 0 for a sphere to 1 for an infinitely thin plate. The platyness $\eta$ is related to the thickness/width aspect ratio $\lambda$ through the simple expression $\eta=1-1 / \lambda$. In the following, $\eta$ is varied systematically from $\eta=0$, which corresponds to spherical particles, to $\eta=0.94$, which corresponds to particles 17 times longer than thick.

\subsection{Simulation method}

We employed the Molecular Dynamics method, as usually called in the context of granular simulations since the pioneering work of Cundal and Strack $[38,39]$. In applying Molecular Dynamics to spheroplates, we should distinguish the contacts between different elements (vertices, edges, and faces) of two interacting particles. Each interaction represents single or multiple contacts, each contact occurring between two elements belonging to either of the two spheroplates. All possible contacts are determined by considering two cases: a contact between two edges and a contact between a vertex and a face [40,31].

As usual in molecular dynamics simulations, normal dissipation is accounted for by viscous damping. The contact forces are calculated using the linear spring-dashpot model and the Coulomb friction law. Then, the interaction forces are calculated by adding the contact forces exerted at each contact point. A detailed description of the contact laws can be found in Ref. [31].

\subsection{Sample construction and shear test}

Twelve monodisperse samples made up of 8000 spheroplates of radius $r$ are built. The difference between these samples is their platyness, which varies from $\eta=0$ to 0.94 . The damping parameter and the normal stiffness (force per unit overlap) were adjusted in order to get largest time step $\left(10^{-6} s\right)$ and small overlaps within numerical stability. The normal stiffness and the interparticle coefficient of friction were $1.5 \times 10^{-9} \mathrm{~N} / \mathrm{m}$ and 0.58 , respectively.

Initially, the particles are placed at the nodes of a cubic grid of side $2 \sqrt{2} R$ and each of them is randomly oriented. Then the samples are isotropically compressed. Once the stic equilibrium is reached, the lateral walls are removed and replaced by periodic boundaries. The samples are then sheared by imposing a constant horizontal velocity $v_{w}$ and a constant confining stress $\sigma_{w}$ to the upper wall allowing for the volume of the sample to vary during the test. The particles in contact with the walls are "glued" to them in order to avoid strain localization at the boundaries. In all simulations presented in this paper the gravity is set to zero in order to get homogeneous stress fields inside the packings.

As mentioned in Sec. 1, the focus of this work is on the microstructural properties of the packings in the steady state. Therefore, the samples are sheared up to a large cumulative shear strain $\gamma=x_{w} / y_{w} \simeq 2.5$, where $x_{w}$ is the horizontal displacement of the upper wall and $y_{w}$ is its vertical position. The shear stresses and velocities are 


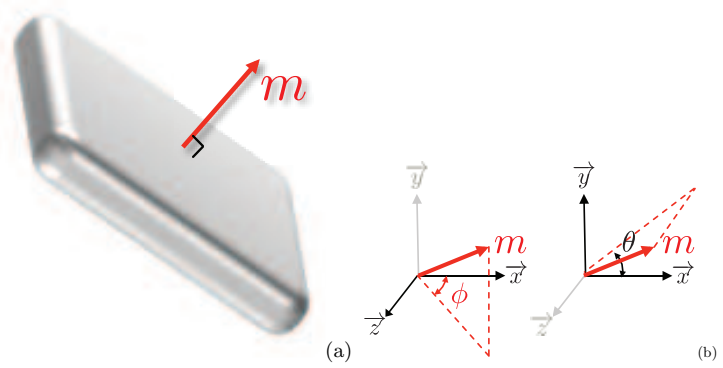

Fig. 2. (a) Definition of the particle orientation vector $\mathbf{m}$ (normal to the particles face). (b) Orientation angles $\theta$ and $\phi$ of the vector $\mathbf{m}$ at the reference system

such that the test is carried out in the quasistatic limit [41]. All packings reach the steady state where both the strength and volume fluctuate around mean values, and the strain is homogeneously distributed in the sample.

\section{Simulation results}

In this section, we present the results of our simulations and analyses. All the data shown in this section represent an average over the last $40 \%$ of cumulative shear strain (i.e., from $\gamma=1.5$ to 2.5 ).

\subsection{Particle alignment and face-face interactions}

The particle orientation is defined as the orientation of the vector $\mathbf{m}$, normal to the particle face; see Fig. 2(a). The distribution of particle orientations can be represented by the probability density function $P_{m}(\Omega)$ of particles whose vector $\mathbf{m}$ is along the solid angle $\Omega=(\theta, \phi)$; see Fig. $2(\mathrm{~b})$. Figure 3 shows $P_{m}(\Omega)$ for all samples at a shear strain $\gamma=2$. We see that, as the particles platyness increases, the distributions become more anisotropic, indicating that the number of particles aligning their faces along a particular direction increases. We also see that, as the particle platyness increases, the priviledged direction of the distributions gradually changes from that of the major principal stress to the vertical direction. It is interesting to note that this type of ordering appears even in the samples composed of particles of very low platyness (e.g., for $\eta=0.14$ ), whose shape deviates only slightly from that of a sphere.

Because of the planar symmetry of our simple shear tests, it is practical to analyze the system in terms of the restriction of $P_{m}(\Omega)$ to the $x y$ plane, i.e., the function $P_{m}(\theta)$, which can be approximated by its lowest order Fourier expansion:

$$
P_{m}(\theta) \simeq 1 / \pi\left[1+a_{m} \cos 2\left(\theta-\theta_{m}\right)\right]
$$

where $a_{m}$ is the anisotropy of particle orientations and $\theta_{m}$ is the principal direction of $P_{m}(\theta)$. Even though the parameters $a_{m}$ and $\theta_{m}$ can be calculated by fitting the measured values of $P_{m}(\theta)$ to the approximation presented

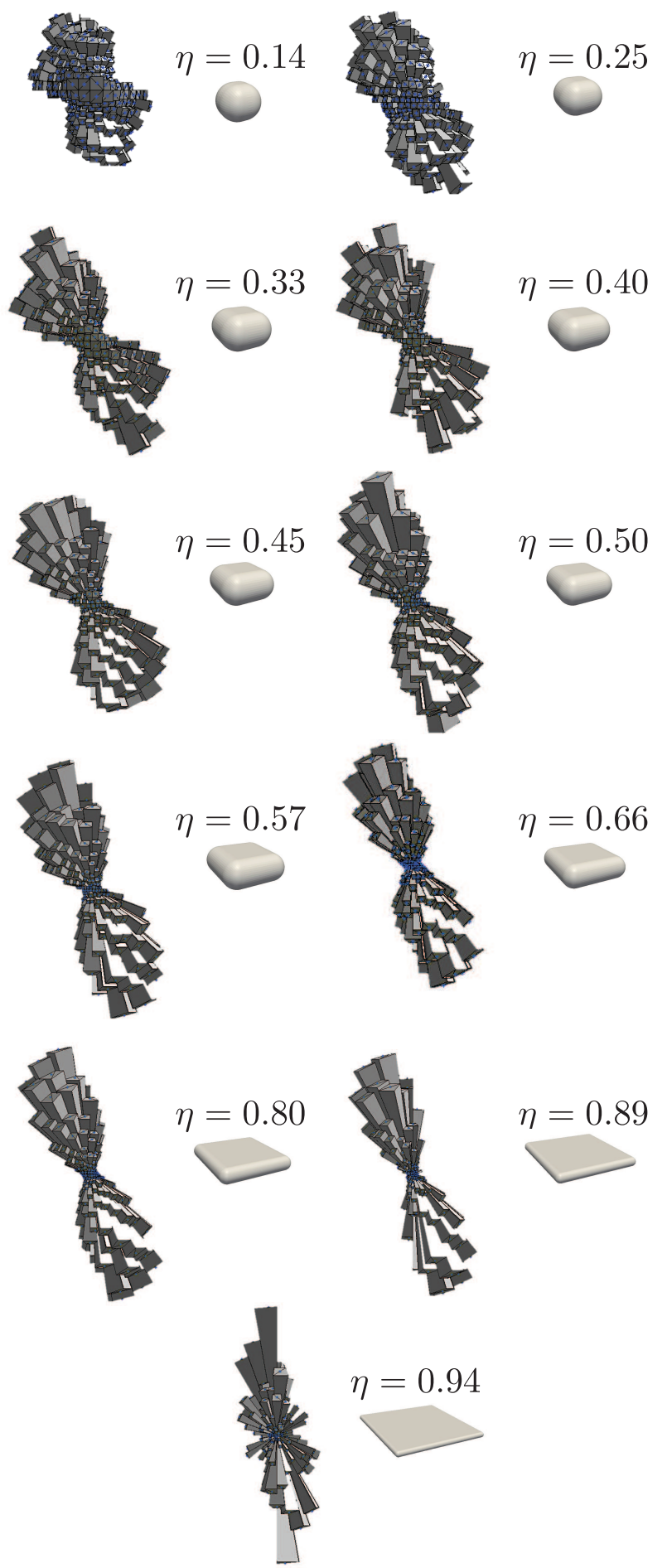

Fig. 3. Probability density functions $P_{m}(\Omega)$ of particle orientations for all values of $\eta$, at shear strain $\gamma=2$ 


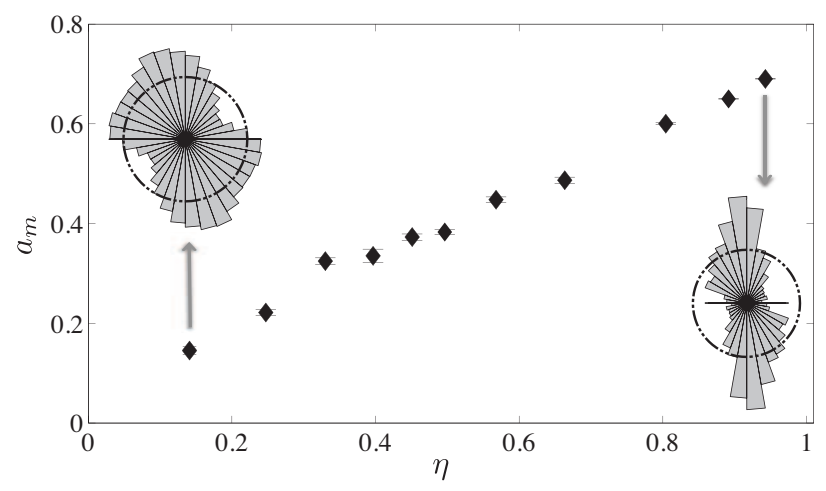

Fig. 4. Anisotropy of particle orientations $a_{m}$ as a function of platyness $\eta$. Error bars indicate the standard deviation

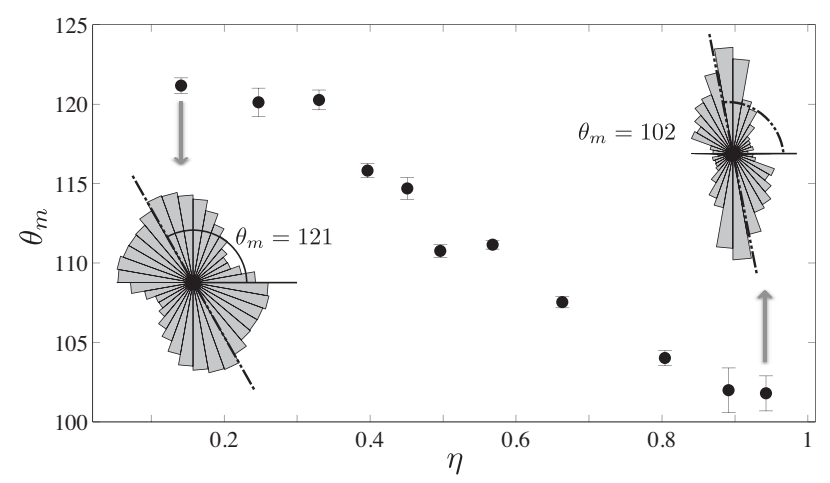

Fig. 5. Principal direction of particle orientations $\theta_{m}$ as a function of $\eta$. Error bars indicate the standard deviation.

in Eq. (2), in practice it is more convenient to calculate these parameters using the nematic tensor defined by

$$
\mathrm{M}=\frac{1}{N_{p}} \sum_{p \in V} m_{\alpha}^{p} m_{\beta}^{p},
$$

where $N_{p}$ is the number of particles $p$ in the volume $V, \mathbf{m}$ is the particle orientation, and $\alpha$ and $\beta$ denote the components in the reference frame. The anisotropy of particle orientations is $a_{m}=2\left(\mathrm{M}_{1}-\mathrm{M}_{3}\right)$ and the principal direction $\theta_{m}$ is that of the first eigenvalue of M.

Figure 4 shows the anisotropy of particle orientations $a_{m}$ as a function of platyness $\eta$. We see that $a_{m}$ increases with $\eta$, from 0 to $\simeq 0.7$, confirming that, as platyness increases, the number of particles aligning their faces also increases. Figure 5 shows the principal direction $\theta_{m}$ as a function of $\eta$. We see that $\theta_{m}$ decreases with $\eta$, approximately from $120^{\circ}$ to almost $100^{\circ}$, confirming that, as platyness increases, the direction along which these particles align gradually changes from that of the major principal stress (i.e., $135^{\circ}$ ) to the vertical direction (i.e., $90^{\circ}$ ).

The fact that an increasing number of particles tend to align their faces along a preferential direction is closely related to one of the distinctive features of materials made up of platy particles: the possibility of forming face-face interactions. Figure 6 shows the proportion $\zeta$ of face-face interactions as a function of $\eta$. We see that $\zeta$ rapidly increases with $\eta$ from 0 to $\simeq 0.15$, confirming that face-face interactions are enhanced by the degree of platyness.

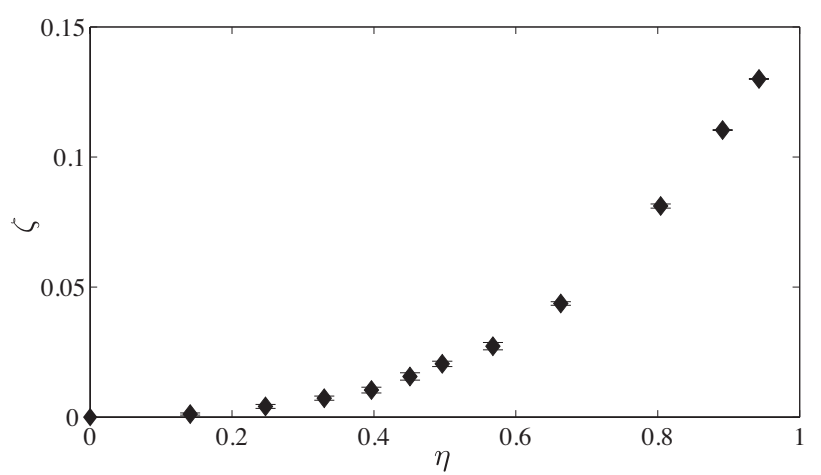

Fig. 6. Proportion $\zeta$ of face-face interactions as a function of platyness $\eta$. Error bars indicate the standard deviation.

\subsection{Local order}

The increase of the proportion of face-face interactions suggests the formation of locally ordered structures characterized by face-face interactions. These structures can be tracked by means of the radial distribution function $g\left(r^{\prime}\right)$ of the radial positions $r^{\prime}$ of particle centers, defined as

$$
g\left(r^{\prime}\right)=\left\langle\frac{N_{p}\left(r^{\prime}+\Delta r^{\prime}\right)}{\rho V_{\Delta r}}\right\rangle_{p}
$$

where $\rho$ is the number of particles per unit volume, $N_{p}\left(r^{\prime}+\right.$ $\left.\Delta r^{\prime}\right)$ is the number of particles whose center of mass is inside a differential volume $V_{\Delta r^{\prime}}$ (defined as a thin shell of internal radius $r^{\prime}$ and external radius $r^{\prime}+\Delta r^{\prime}$ ), and the average is computed using all the particles $p$ in the system.

Figure 7 shows $g\left(r^{\prime}\right)$ for all values of $\eta$. For the sphere packing, i.e., for $\eta=0$, shown in Fig. 7(a), the local order is characterized by peak values representing successive shells of neighboring particles as illustrated by the schemes inside the figure. At low values of $\eta$, shown in Figs. 7 (a) and (b), the peaks of $g\left(r^{\prime}\right)$ gradually vanish as $\eta$ increases. This means that, as platyness increases, shell structures disappear due to the aspherical shape of the particles. This tendency goes on to the point where there are almost no peaks in the function $g\left(r^{\prime}\right)$, e.g., see $g\left(r^{\prime}\right)$ for $\eta=0.57$ and $\eta=0.66$. For the systems with still higher values of $\eta$, shown in Fig. 7(c), the first peak of $g\left(r^{\prime}\right)$ reappears, showing the emergence of a new kind of local order.

In order to characterize this evolution of local order with platyness, we plot in Fig. 8 the height $g_{\max }$ of the first peak as a function of $\eta$. The height declines from $g_{\max } \simeq 6$ for $\eta=0$ down to $g_{\max } \simeq 1.1$ for $\eta=0.66$, then it increases again for $\eta>0.66$. Note that in this range the first peak occurs at the same distance $r^{\prime} \simeq 2 r$. Since this distance can only occur for face-face interactions, the new local structures are mainly composed of face-face interactions as shown in the schemes in Fig. 7(c).

\subsection{Cluster formation}

Both the increase of the number of face-face interactions and the nonlinear evolution of the pair correlation func- 

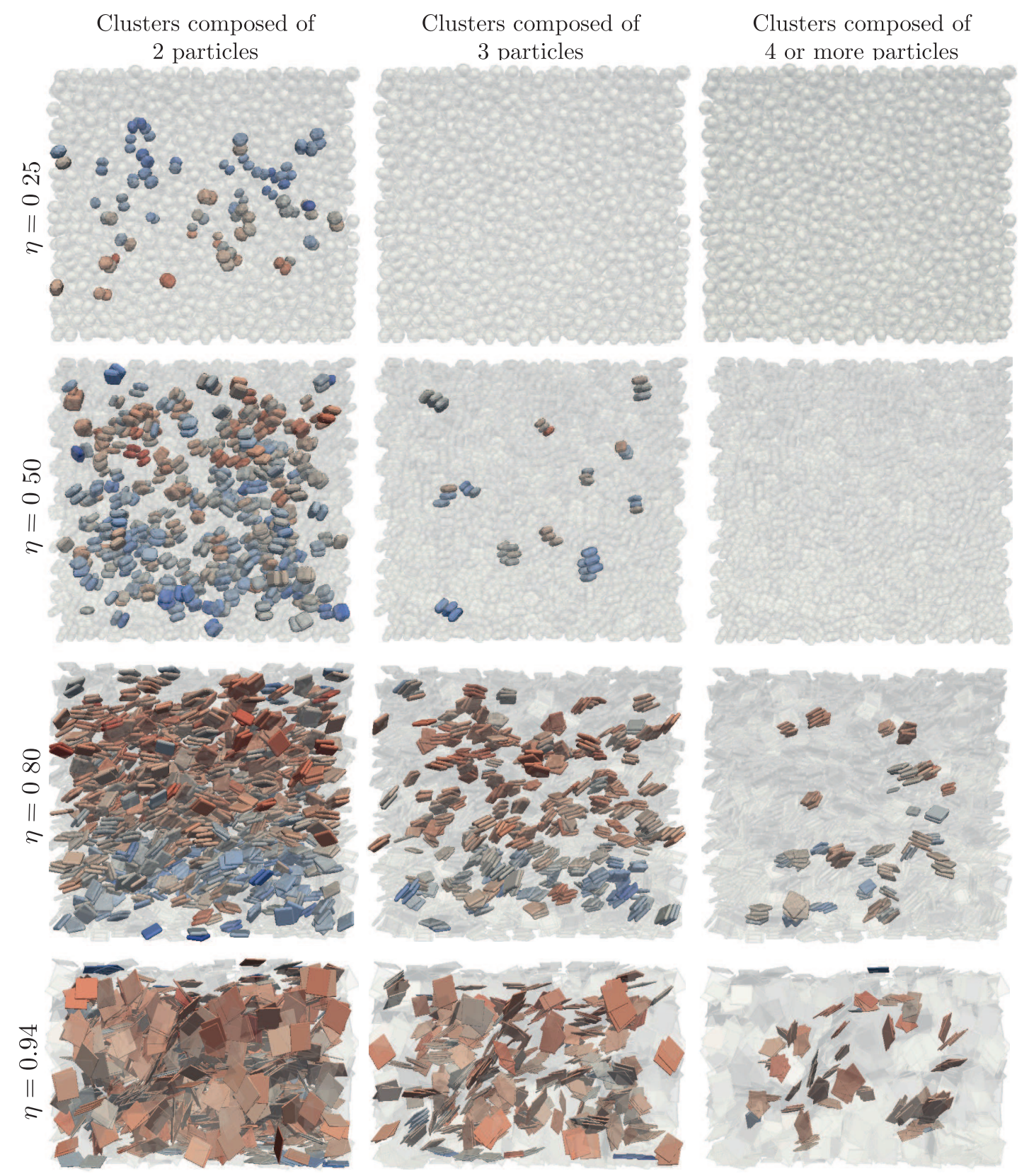

Fig. 9. Sideview of clusters composed of two, three, and four or more particles in four samples with platyness $\eta=0.25,0.50$, 0.80 , and 0.94 . Colors indicate the relative angular velocity, where cold and hot colors represent static and rotating clusters respectively.

tion suggest that higher-order correlations in the form of clusters should appear in the system at high degree of platyness. Such clusters may be identified by considering the particles connected by a face-face contact. All such particles are defined to belong to the same cluster, to which we refer below as a "face-connected" cluster. Fig. 9 shows snapshots of all clusters composed of two, three, and four or more particles, in four samples with $\eta=0.25$, $0.50,0.80$, and 0.94 . At low particle platyness, we observe mainly clusters of two particles whereas at higher platyness larger clusters are observed.
Fig. 10 displays the total number $N_{c}$ of clusters and the mean number $\langle S\rangle$ of particles per cluster as a function of $\eta$. Both $N_{c}$ and $\langle S\rangle$ increase continuously with $\eta$. In other words, as platyness increases, not only more face-connected clusters appear but the clusters are also increasingly larger. $\langle S\rangle$ grows from 2 at lowest platyness to 2.4 for our largest platyness. This rather low value reflects the fact that there are many more clusters composed of $S=2$ particles than clusters of higher order.

In order to get insight into the nature of such clusters, it is useful to consider the proportion $n$ of clusters composed of $S$ particles, as in the precolation theory (where 

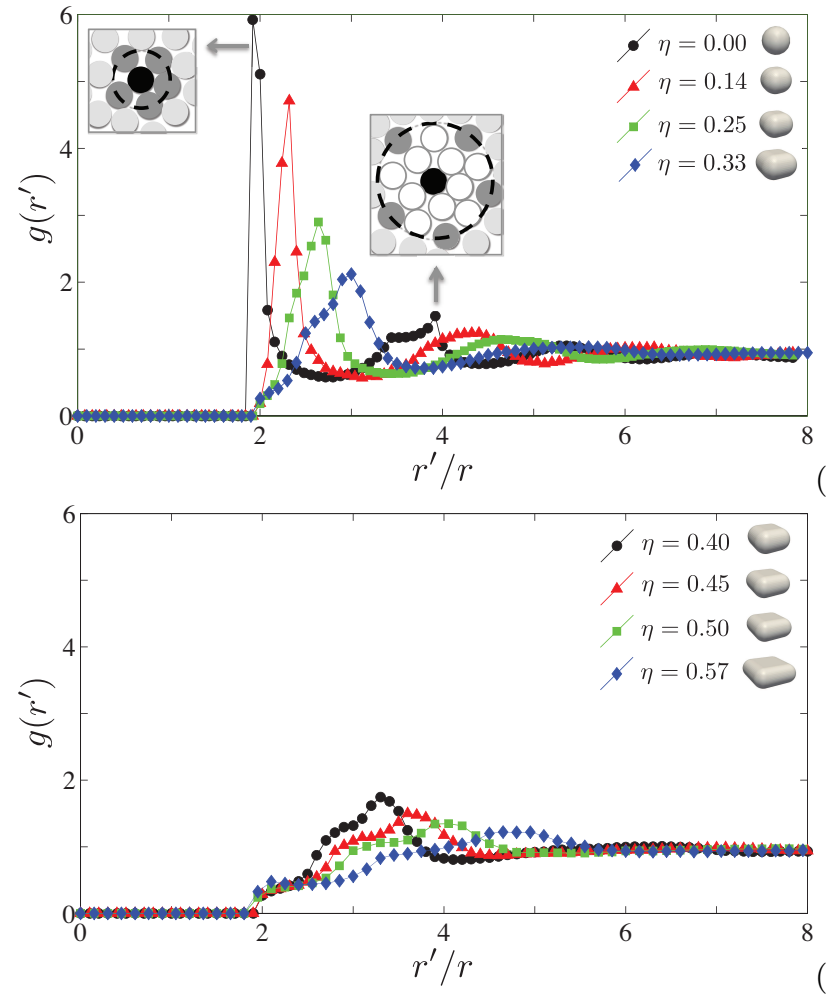

(a)

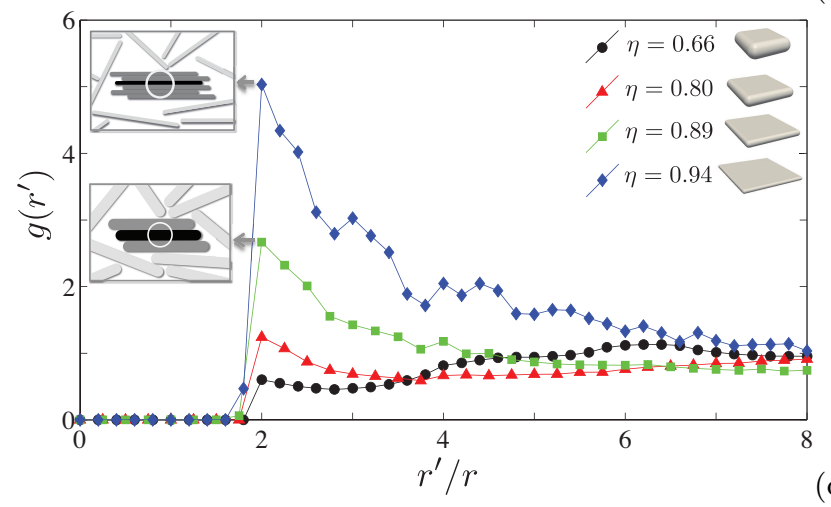

(b)

Fig. 7. Radial distribution functions $g\left(r^{\prime}\right)$ of the radial positions $r^{\prime}$ of the particle centers, for: (a) particle platyness $\eta$ between 0 and 0.33 , (b) $\eta$ between 0.40 and 0.57 , and (c) $\eta$ between 0.66 and 0.94 .

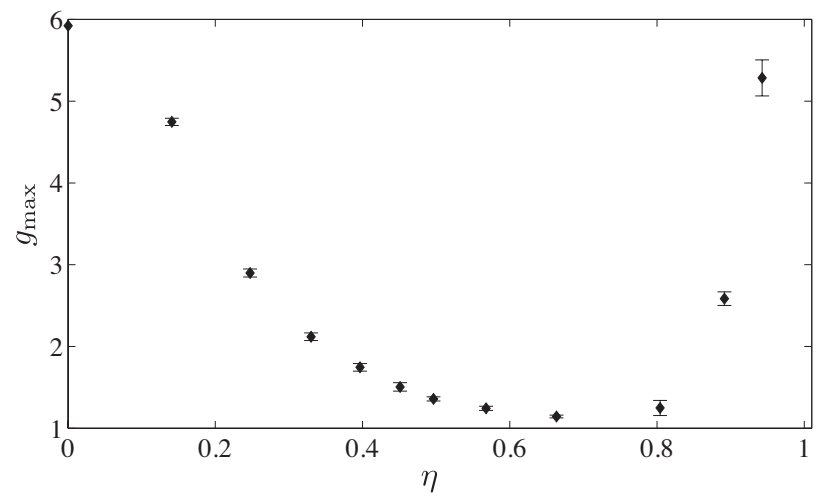

Fig. 8. Height $g_{\max }$ of the first peak of pair correlation functions as a function of platyness $\eta$. Error bars indicate the standard deviation.
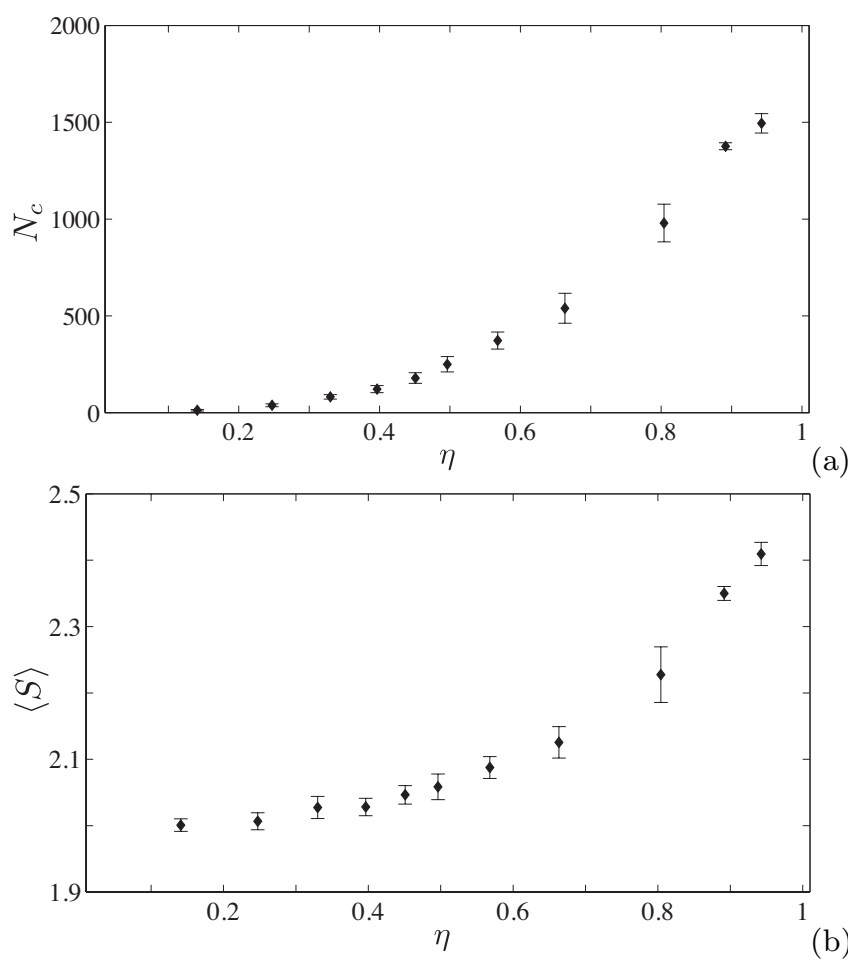

Fig. 10. (a) Total number of clusters $N_{c}$ and (b) mean number $\langle S\rangle$ of particles per cluster, as functions of $\eta$. Error bars indicate the standard deviation.

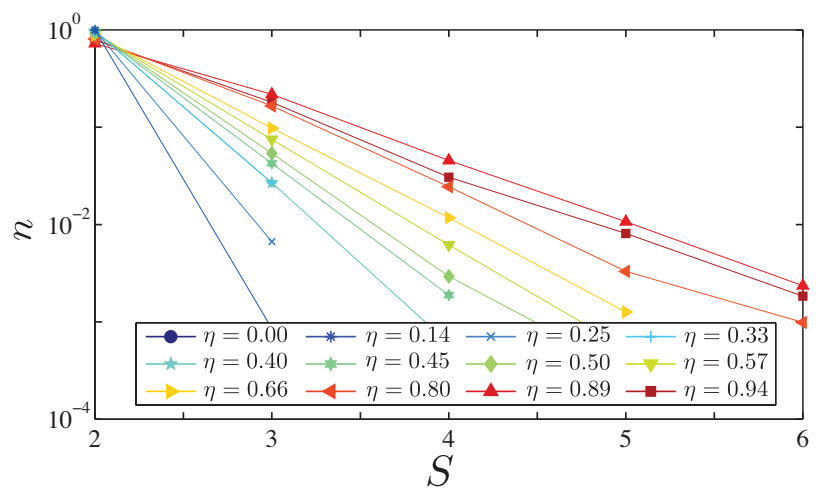

Fig. 11. Proportion $n$ of clusters composed of $S$ particles as a function of $S$ for all values of $\eta$.

the particles represent the sites and the face-face contacts are the bonds). Figure 11 displays $n(S)$ for $S=2,3$, 4,5 and 6 . It is interesting to see that, for all values of platyness with at least three data points, the number of clusters declines exponentially with $S$. This behavior is consistent with the bond random percolation model well below the percolation threshold [?]. Actually, by inspecting Fig. 9, we see that the high-order clusters (i.e., clusters with $S \geq 4$ ) are quite rare and hence even at high platyness the system is far from the percolation of face-face bonds. 


\section{Summary and discussion}

In summary, we have analyzed the structural properties and the particle clustering of sheared packings composed of platy particles, by means of molecular dynamic simulations. The platyness was varied from 0 , corresponding to a sphere, to 0.94 for particles that are 17 times longer than thick. The samples were sheared up to a large shear deformation and analyzed in the steady state.

Our results indicate that particle platyness enhances the spontaneous alignment of the particle faces. Due to dynamics in continuous shearing, this ordering phenomenon emerges even in systems composed of particles with very low platyness with a shape differing only slightly from spherical shape. This kind of ordering has previously been observed in experiments $[27,22]$ and numerical simulations mainly with two-dimensional elongated particles $[28,15$, 18]. Regarding the privileged direction of alignment, we found that, as platyness increases, this direction gradually changes from that of the major principal stress to a direction that is perpendicular to the shear direction. In other words, the larger axis of the particles tends to align with the average velocity field. This evolution of the privileged direction of alignment is consistent with several observations reported in Refs. $[28,27,22]$.

The occurrence of face-face interactions may be attributed to this alignment of the particles due to continuous shearing. The increase of these interactions has also been observed in experiments [42] as well as in numerical simulations with elongated particles in $2 \mathrm{D}[15,18]$.

By analyzing the radial distribution functions of particle positions, we evidenced a transition between two regimes characterized by different microstructures. In the first regime at low values of platyness, the platy shape of the particles disturbs the usual shell structure of spherical particles by enhanced steric hindering between particles. In contrast, in the second regime at high values of platyness, the platy shape enhances local order characterized by face-face interactions. The orientational and positional orders evidenced in these systems are analogous to those observed in liquid crystals. The first regime is mainly characterized by orientational order, which is reminiscent of nematic order, whereas in the second regime a positional "smectic" order emerges in the form of face-face interactions.

Finally, we showed that the random occurrence of such interactions leads to the formation of face-connected clusters with exponentially decaying size. But even at high platyness, the proportion of face-face interactions is too low to allow for percolating clusters throughout the system. In addition, it is also surprising that these clusters emerge here uniquely as a consequence of particle shape even in the absence of attraction forces between the particles, even if it is obvious that they should be more stable and reinforced in the presence of attraction forces as is the case in clays [1]. This is the goal of our ongoing investigation.

We acknowledge financial support by the Ecos-Nord program (Grant No. C12PU01).

\section{References}

1. J. Mitchell and K. Soga, Fundamentals of Soil Behavior (Wiley, New York, 2005)

2. J. Santamarina, Soils and Waves (Wiley \& Sons, New York, 2009)

3. A. Anandarajah, Comput. Geotech 27, (2000) 10.

4. A. Mirghasemi, L. Rothenburg and E. Maryas, Geotechnique, 52 (2002) 209.

5. K. Mair, J. Geophys. Res. 107, (2002) 2219.

6. G. Cho, J. Dodds, and J. Santamarina, J. Geotech. Geoenviron. Eng. 132, (2006) 591.

7. E. Azéma and F. Radjai and R. Peyroux and G. Saussine, Phys. Rev. E 76, (2007) 011301.

8. S. A. Galindo-Torres, F. Alonso-Marroquin, Y. C. Wang, D. Pedroso, and J. D. Munoz Castano, Phys. Rev. E 79, (2009) 060301.

9. R.-C Hidalgo, I. Zuriguel, D. Maza, and I. Pagonabarraga, Phys. Rev. Lett 103, (2009) 118001.

10. A. Donev, F. H. Stillinger, P. M. Chaikin, and S. Torquato, Phys. Rev. Lett. 92, 255506 (2004).

11. A. Donev, I. Cisse, D. Sachs, E. Variano, F. Stillinger, R. Connelly, S. Torquato, and P. Chaikin, Science 303, (2004) 990.

12. W. Man, A. Donev, F.-H. Stillinger, M. T. Sullivan, W.-B. Russel, D. Heager, S. Inati, S. Torquato, and P. M. Chaikin, Phys. Rev. Lett. 94, (2005) 198001.

13. I. Zuriguel and T. Mullin, Proc. R. Soc. A 464, (2008) 99.

14. T. Kanzaki, M. Acevedo, I. Zuriguel, I. Pagonabarraga, D. Maza, and R.C. Hidalgo2, Eur. Phys. J. E. 34, (2011) 133

15. E. Azéma and F. Radjai, Phys. Rev. E 81, (2010) 051304.

16. E. Azéma, N. Estrada and F. Radjai, Phys. Rev. E 86, (2012) 041301.

17. CEGEO, B. Saint-Cyr, K. Szarf, C. Voivret, E. Azéma, V. Richefeu, J.-Y. Delenne, G. Combe, C. Nouguier-Lehon, P. Villard, P. Sornay, M. Chaze and F. Radjai EPL, 98 (2012) 44008

18. E. Azéma and F. Radjai, Phys. Rev. E 85, (2012) 031303. 19. E. Azéma, F. Radjai and F. Dubois, Phys. Rev. E 87, (2013) 062203.

20. B. Saint-Cyr, J.-Y. Delenne, C. Voivret, F. Radjai, and P. Sornay, Phys. Rev. E 84, 041302 (2011).

21. E. Azéma, F. Radjai, B. Saint-Cyr, J.-Y. Delenne, and P. Sornay, Phys. Rev. E 87, 052205 (2013).

22. T. Borzsonyi and R. Stannarius, Soft Matter 9, (2013) 7401-7418.

23. G.Lumay and and N.Vandewalle, Phys.Rev.E, (2006), 74, 021301.

24. K. Anki Reddy, V. Kumaran and J. Talbot, Phys. Rev. E, 80, (2009) 031304.

25. C.S.Campbell, Phys.Fluids, 23, (2011) 013306.

26. P.W.Cleary, PowderTechnology, 179, (2008) 144.

27. T. Borzsonyi, B. Szabo, G. Toros, S. Wegner, J Torok, E. Somfai, T. Bien, and R Stannarius, Phys. Rev. Lett. 108, 2012228302

28. A. A. Pena, R. Garcia-Rojo, and H. J. Herrmann, Granular Matter 9, 279 (2007).

29. L. Pournin, M. Weber, M. Tsukahara, J.-A. Ferrez, M. Ramaioli, and T. M. Liebling, Granular Matter 7, 119 (2005).

30. F. Alonso-Marroquin, Europhys. Lett. 83, (2008) 14001.

31. M. Boton, E. Azéma, N. Estrada, F. Rdajai and A. Lizcano, Phys.Rev. E 87, (2013) 032206.

32. A. Anandarajah, Eng. Geol. 47, (1997) 313. 
33. A. Anandarajah, Comput. Geotech. 27, 1 (2000).

34. M. Yao and A. Anandarajah, J. Eng. Mech. 129, (2003) 585.

35. P. A. Langston, M. A. Al-Awamleh, F. Y. Fraige, and B. N. Asmar, Chem. Eng. Sci. 59, (2004) 425.

36. F. Alonso-Marroquin and Y. Wang, Granular Matter 11, 317 (2009).

37. S. A. Galindo-Torres, D. Pedroso, D. J. Williams, and L. Li, Comput. Phys. Commun. 183, 266 (2012).

38. F. Radjai and F. Dubois, Discrete-Element Modeling of Granular Materials (John Wiley \& Sons, New York, 2011)

39. P. A. Cundall and O. D. L. Strack, Géotechnique 29, (1979) 47.

40. J. Ghaboussi and R. Barbosa, Int. J. Num. Anal. Methods Geomech. 14, (1990) 451.

41. GDR MiDi, Eur. Phys. J. E 14, (2004) 341.

42. M. Acevedo, R. C. Hidalgo, I. Zuriguel, D. Maza, and I. Pagonabarraga, Phys. Rev. E 87, (2013) 012202. 